\title{
The Caspase Pathway in Noise-Induced Apoptosis of the Chinchilla Cochlea
}

\author{
Thomas M. Nicotera, ${ }^{1}$ Bo Hua Hu, ${ }^{2}$ and Donald Henderson ${ }^{2}$ \\ ${ }^{1}$ Molecular and Cellular Biophysics, Roswell Park Cancer Institute, Buffalo, NY 14263, USA \\ ${ }^{2}$ Center for Hearing and Deafness, State University of New York at Buffalo, Buffalo, NY 14214, USA
}

Received: 2 August 2002; Accepted: 18 April 2003; Online publication: 13 October 2003

\begin{abstract}
We previously reported that intense noise exposure causes outer hair cell (OHC) death primarily through apoptosis. Here we investigated the intracellular signal pathways associated with apoptotic OHC death. Chinchillas were exposed to a $4 \mathrm{kHz}$ narrowband noise at $110 \mathrm{~dB}$ SPL for $1 \mathrm{~h}$. After the noise exposure, the cochleas were examined for the activity of each of three caspases, including caspase- 3 , -8 , or -9 with carboxyfluorescein-labeled fluoromethyl ketone (FMK)-peptide inhibitors. The cochleas were further examined for cytochrome c release from mitochondria by immunohistology and for DNA degradation by the TUNEL method. The results showed that the noise exposure triggered activation of caspase- 3 , an important mediator of apoptosis. The noise exposure also caused the activation of caspase- 8 and caspase-9, each of which is associated with a distinct signaling pathway that leads to activation of caspase-3. Caspase activation occurred only in the apoptotic OHCs and not in the necrotic OHCs. These results indicate that multiple signaling pathways leading to caspase-3 activation take place simultaneously in the apoptotic OHCs. In addition to caspase activation, noise exposure caused the release of cytochrome c from mitochondria, resulting in a punctate fluorescence in the cytosol. In contrast to activation of caspases, the release of cytochrome $\mathrm{c}$ took place in both apoptotic and necrotic OHCs. Moreover, the release of cytochrome $\mathrm{c}$ in a subpopulation of OHCs took place
\end{abstract}

Correspondence to: Thomas M. Nicotera - Roswell Park Cancer Institute $\cdot$ Molecular and Cellular Biophysics $\cdot$ Elm \& Carlton Streets - Buffalo, NY 14263 - Telephone: (716) 845-8294; fax: (716) 8458899; email: thomas.nicotera@ roswellpark.org early in the cell death process, prior to any outward signs of necrosis or apoptosis. These data suggest that in this subpopulation there exists a common step that is shared by cell death pathways before entering either necrosis or apoptosis. Lastly, use of the TUNEL assay in combination with PI labeling provides a more accurate discrimination between apoptosis and necrosis.

Keywords: noise, apoptosis, caspase, cytochrome c, outer hair cell, chinchilla

\section{INTRODUCTION}

Hair cell (HC) damage induced by exposure to intense noise has been observed in the organ of Corti for a long time (Henderson and Hamernik 1986; Lim and Melnick 1971; Omata and Schatzle 1981). Microscopic examination of the organ of Corti shows diverse morphological changes in the damaged HCs. The diversity of morphologic changes suggests involvement of different pathological mechanisms in the progression of cell death. Recent studies have indicated that both apoptosis and necrosis are implicated in the cochlear lesion after exposure to intense noise ( $\mathrm{Hu}$ et al. 2000; Nicotera et al. 2002). Apoptosis is associated with an active cell death process featured morphologically by nuclear condensation and fragmentation, whereas necrosis is a passive consequence of gross injury to cells and marked by nuclear swelling (Lo et al. 1995; Majno and Joris 1995). In noise-damaged regions within the organ of Corti, apoptotic HCs are manifest primarily in those sections receiving 
maximal sound stimuli. In contrast, necrotic cells are spread out over a large area of the organ of Corti $(\mathrm{Hu}$ et al. 2000). It has been reported that apoptosis is the major pathway by which expansion of the cochlear lesion takes place in the chinchilla after exposure to an intense noise (Hu et al. 2002a).

Apoptosis is a tightly controlled and well-organized physiological process for removal of unwanted or unneeded cells. Apoptotic signaling can take place through multiple independent pathways that are initiated by triggering events either from within or outside the cell. In either case, the triggering pathways converge on a common mechanism for the internal degradation of cells that is initiated by the caspase family of proteases. These signaling pathways that direct cells to undergo apoptosis are currently under intensive investigation (Chen and Tan 2000; Maher and Schubert 2000; Strasser et al. 2000).

Caspases are a family of aspartate-specific cysteine proteases, which exist as latent intracellular zymogens (Miller 1997; Nicholson and Thornberry 1997). To date, at least 14 caspases have been identified and are divided into two groups; apoptotic initiators (caspases$2,-8,-9$, and -10) and apoptotic effectors (caspases-3, -6 , and -7 ), based on their putative functions and sequence homologies (Eldadah and Faden 2000). Once activated, effector caspases selectively cleave distinct intracellular substrates that lead to dismantling of a cell's architecture, signaling apparatus, and repair mechanisms (Takahashi 1999). The sequence of caspase activation shows that distinct cascades are activated depending on the specific pathology, conditions employed, and cell type examined.

Caspase-3 is the major apoptotic effector protease whose activation involves one of several upstream initiator caspases, namely, caspase- 8 or caspase- 9 , that represent two distinct apoptotic signal transduction pathways. Caspase- 8 is involved in the extrinsic apoptotic pathway, which is initiated following the binding and trimerization of death receptors localized on the cell membrane. Once activated, caspase- 8 can activate downstream caspases by direct cleavage or indirectly by cleaving Bid and inducing cytochrome c release from mitochondria. In contrast, caspase-9 is involved in an intrinsic pathway associated with mitochondria-mediated activation and subsequent release of cytochrome $\mathrm{c}$ to the cytosol (Adrain and Martin 2001). Oligomerization of cytochrome c with two other cytosolic protein factors, Apaf-1 and pro-caspase 9, results in the activation of procaspase 3. Cytochrome $\mathrm{c}$ is an essential component of the mitochondrial respiratory chain whose function is to transport electrons from the coenzyme QH2-cytochrome c reductase complex to the cytochrome c oxidase complex in the electron transport chain (Hatefi 1985).
TABLE 1

Number of animals used for each treatment after noise exposure

\begin{tabular}{lcccc}
\hline & Immediately & 6-hour & 2-day & Control \\
\hline Caspase-3 & 5 & 2 & 3 & 3 \\
Caspase-8 & 3 & 0 & 0 & 3 \\
Caspase-9 & 3 & 0 & 0 & 3 \\
Cytochrome c & 4 & 0 & 0 & 2 \\
TUNEL & 3 & 0 & 0 & 2 \\
\hline
\end{tabular}

The signaling pathways that control the initiation and execution of apoptosis in noise-damaged HCs are just beginning to be understood. Pirvola et al. (2000) reported that the c-Jun-N-terminal kinase (JNK), also known as the stress-activated protein kinase (SAPK), is associated with processing of noise-induced apoptosis of HCs. JNK is activated through its phosphorylation and has been shown to be important in neuronal cell death responses. Inhibition of c-Jun phosphorylation and consequent activation protect both neuronal cells and HCs from apoptosis (Pirvola et al. 2000). We have reported that the activation of caspase- 3 takes place in apoptotic HCs immediately after a noise exposure, thus indicating its participation in noise-induced apoptosis (Hu et al. 2002a). Information regarding the activation of downstream caspase- 3 and its relationship to the JNK are as yet unknown.

This study was designed (1) to expand the current knowledge on caspase- 3 activation in apoptotic outer hair cells (OHCs) by examining the caspase- 3 activity at various times of the noise exposure; (2) to explore which initiator caspases, caspase-8, -9 , or both, are activated after the noise exposure; (3) to detect the release of cytochrome $\mathrm{c}$ from mitochondria to the cytosol in the noise-damaged OHCs; and (4) to improve the methodology to better distinguish between apoptosis from necrosis. Knowledge of the signaling pathways responsible for regulating the apoptotic processes is important in light of the potential for developing therapeutic strategies to either reverse or prevent apoptosis.

\section{MATERIALS AND METHODS}

\section{Subjects}

A total of 32 adult chinchillas (450-600 g) were used in this study. Each animal was exposed to noise as described in the following section. Chinchillas were processed for detection of activated caspases-3, -8, or -9 , the release of cytochrome c, or DNA degradation following a noise exposure. The number of animals used for each experimental condition is depicted in Table 1. Five animals without exposure to the noise 
were used as negative controls for the fluorescent labeling protocol.

\section{Noise exposure}

The narrowband noise employed in these experiments was centered at $4 \mathrm{kHz}$ and generated using a $\mathrm{D} / \mathrm{A}$ converter on a signal processing board (Loughborough TMS 32020) that was controlled by a personal computer (IBM compatible). The noise signal was routed through an attenuator (HP $350 \mathrm{D})$, a filter (Krohn-Hite 3550R), and a power amplifier (NAD 2200) and finally to an acoustic horn (JBL 2360). The loudspeaker was suspended directly above the cages. Noise levels were measured using a sound level meter (Larson and Davis 800B), connected to a preamplifier (Larson and Davis model 825) and a condenser microphone (Larson and Davis, LDL 2559). The microphone was positioned within the cage at the level of the animal's head. Each animal was exposed to the noise at a level of $110 \mathrm{~dB}$ SPL for 1 $\mathrm{h}$. Animals were housed in a quiet animal colony when not being exposed to the noise.

\section{Detection of active caspases-3, -8 , and -9}

Caspases-3, -8, and -9 were detected using three fluorescent probes, FAM-DEVD-FMK, FAM-LETD-FMK, and FAM-LEHD-FMK, respectively (Intergen Company, Purchase, NY). The methodology is based on carboxyfluorescein-labeled fluoromethyl ketone (FMK)-peptide inhibitors of caspases. These inhibitors are cell permeable and noncytotoxic. Once inside the cell, the inhibitor irreversibly binds to the active caspase and fluoresces in association with the active caspase.

The basic labeling procedures for detecting each of the three caspases are similar. The animal was anesthetized with a mixture of ketamine $(35 \mathrm{mg} / \mathrm{kg})$ and acepromazine $(0.5 \mathrm{mg} / \mathrm{kg})$ at a defined time after the noise exposure. Both the left and right cochleas were surgically exposed using a conventional ventral approach. Two small openings were drilled in the bony shell over the scala vestibuli and the scala tympani in the basal turn of the cochlea. The cochleas were perfused with approximately $30 \mu \mathrm{l}$ of the freshly prepared solutions containing each of three caspase inhibitors (caspases-3, -8 , and -9 were diluted according to the manufacturer's instructions) and incubated for $1 \mathrm{~h}$. The cochleas were then perfused with the manufacturer-provided buffer, followed by the manufacturer-provided fixative. The animals were sacrificed and the cochleas harvested. The cochleas were then dissected in $0.1 \mathrm{M}$ phosphate buffered saline (PBS) and the organs of Corti collected for analysis.

\section{Detection of cytochrome c}

Four chinchillas were used to detect the release of cytochrome c from mitochondria in the noise-damaged OHCs. Chinchillas were sacrificed immediately after noise exposure and the cochleas were quickly removed from the skull and fixed with $10 \%$ buffered formalin for $4 \mathrm{~h}$. The cochleas were then dissected in $0.1 \mathrm{M}$ PBS and the organs of Corti were collected and transferred into a blocking solution Blocker $^{\mathrm{TM}} \mathrm{Ca}-$ sein in PBS; Pierce Chemical Company, Rock Ford, IL) for $60 \mathrm{~min}$ at room temperature. Afterwards, the tissues were rinsed in $0.1 \mathrm{M}$ PBS and permeabilized with $0.2 \%$ Triton X-100 in PBS for $30 \mathrm{~min}$. After a second rinse in PBS, they were incubated with primary antibody (anti-cytochrome c, 6H2.B4; BD PharMingen, San Diego, CA) at a concentration of $2.5 \mu \mathrm{g} / \mathrm{ml}$ in the blocking solution for $1 \mathrm{~h}$ at room temperature and again rinsed. The cochlear tissue was then incubated with primary antibody (Cy2-conjugated) goat anti-mouse IgG, 1: 200; Jackson ImmunoResearch Laboratories, Inc., West Grove, PA) for $1 \mathrm{~h}$, followed by washing and storage in PBS.

\section{Nuclear staining of $\mathrm{OHCs}$ with propidium iodide $(\mathrm{PI})$}

In order to confirm whether a particular process is associated with an apoptotic or necrotic pathway requires that multiple techniques be applied in tandem. Consequently, the morphological changes associated with nuclei were coordinated with cytoplasmic changes in either caspase activity, cytochrome c release into the cytoplasm or DNA fragmentation. Specimens were labeled for either active caspase, cytochrome c, or TUNEL reagents, and then double stained with PI, a DNA intercalating fluorescent probe used for visualization of OHC nuclei. Cochleas were incubated for $10 \mathrm{~min}$ in a $5 \mu \mathrm{g} / \mathrm{ml}$ of PI (Molecular Probe, Inc., Eugene, OR) in 0.1 M PBS, rinsed in PBS, and mounted on slides containing antifade medium (ProLongTM Antifade kit, Molecular Probes).

\section{TUNEL assay for DNA fragmentation}

Nuclear DNA fragmentation was detected using the TUNEL assay (Molecular Probes, Inc). The TUNEL labeling procedure was conducted according to the manufacturer's recommendation with slight modification. Two separate controls were included, one animal was treated with TUNEL reagents but without a noise exposure, while a second animal was exposed to noise and TUNEL staining but without DNA-labeling solution (TdT enzyme and BrdUTP). 
The animals were sacrificed immediately after exposure to noise, followed by removal of the cochleas and fixation for $1 \mathrm{~h}$ with $10 \%$ buffered formalin. The cochleas were then dissected in PBS and the organs of Corti collected. Tissue derived from the two organs of Corti from each animal was processed as a unit. Each tissue was transferred to $1 \mathrm{ml}$ of ice-cold $70 \%(\mathrm{v} / \mathrm{v})$ ethanol and stored in the solution overnight at $-20^{\circ} \mathrm{C}$. The tissue was washed once with manufacturer-provided washing buffer, transferred into the freshly prepared DNA-labeling solution containing $10 \mu \mathrm{l}$ of reaction buffer, $0.75 \mu \mathrm{l}$ of TdT enzyme, $8.0 \mu \mathrm{l}$ of BrdUTP, and $31.25 \mu \mathrm{l}$ of $\mathrm{dH}_{2} \mathrm{O}$, and incubated for $16 \mathrm{~h}$ at room temperature. The tissue was washed twice with manufacturer-provided rinse buffer and then stained with the Alexa Fluor 488 dyelabeled anti-BrdU antibody in the rinse buffer ( $5 \mu \mathrm{l}$ of antibody plus $95 \mu \mathrm{l}$ of rinse buffer) at room temperature for $1 \mathrm{~h}$. The tissue was counterstained with 0.5 $\mathrm{ml}$ of the PI/RNase solution at room temperature for $20 \mathrm{~min}$ and the labeled organs of Corti were mounted on the slide containing antifade medium (ProLongTM Antifade kit, Molecular Probes).

\section{Fluorescence and confocal microscopy}

Representative changes in the organ of Corti were imaged by laser confocal microscopy (Bio-Rad, MRC 1024) after inspection under a fluorescence microscope. Cochleas were double labeled with red fluorescence (PI-labeled nuclei) and green fluorescence (carboxyfluorescein-labeled caspase inhibitor, Cy2labeled cytochrome c, or Alexa Fluor 488 anti-BrdU antibody) and the cochlear section of interest was scanned. A series of approximately 20 laser confocal scanning photographs were taken for each section of the organ of Corti beginning at the top of the OHC stereocilia and lowered to the level of the basilar membrane at depth intervals of $1.0 \mu \mathrm{m}$. Individual and composite images were analyzed using a 3-dimensional image processing software (ImageJ, Wayne Rasband, NIH). Processing of images sequentially, by starting at the top of the hair cell and continuing vertically to the bottom, at $1.0 \mu \mathrm{m}$ increments facilitates the precise 3-dimensional localization of fluorescent events within individual hair cells. All the images presented were projected in a conventional top-down view, except for Figure 6C which is presented as a side-view image. The side-view image was generated electronically by slicing the complete set of images in the $z$ axis using ImageJ software. This approach provided a detailed spatial correlation between the cytosolic accumulation of cytochrome $\mathrm{c}$ and the corresponding nuclear morphology within a particular hair cell.
A previous study demonstrated that 3-dimensional confocal analysis enables us to exclude most nearby supporting cell nuclei from projected images except in severely damaged regions of the organ of Corti. At these severely damaged centers, OHC and supporting cell nuclei can migrate from their original location and yield confounding information (Hu et al. 2002a). In order to avoid confusion in cellular colocalization experiments, most of the images presented were obtained from less damaged cochlear sections.

The care and use of the animals reported in this study were approved by the State University of New York at Buffalo Institutional Animal Care and Use Committee.

\section{RESULTS}

\section{The staining of normal cochleas}

All cochleas were double labeled with PI (red fluorescence) for $\mathrm{OHC}$ nuclei and probed for each of caspases- $3,-8$, and -9 or cytochrome c release (green fluorescence). Little or no fluorescence was observed for any of the three caspases in the control cochleas (not exposed to noise).

Cytochrome c normally resides in the inner membrane space of mitochondria and can be localized using a cytochrome c antibody with a fluorescence tag. In OHCs, mitochondria are distributed primarily along the inner surface of the plasma membrane and in the subnuclear region. In normal cochlea there is a weak fluorescent ring along the cell membrane (Fig. 1) and a second fluorescent cluster in the subnuclear region of OHCs where the mitochondria reside (not present in the image). This fluorescent pattern is similar to that observed in viable OHCs of noise-exposed cochleas.

Both OHCs and inner hair cells exhibited both apoptotic and necrotic changes and both cell types share similar fluorescence patterns for caspases, TUNEL, and cytochrome c. However, since OHCs are considerably more vulnerable to noise exposure than inner hair cells, we described the death processes only in OHCs following a noise exposure.

\section{Detection of activated of caspase-3}

Cochleas exposed to a $4 \mathrm{kHz}$ narrowband noise demonstrated variable $\mathrm{OHC}$ damage in the organ of Corti. The section of the organ of Corti exhibiting maximal damage was confined to a region approximately 6-11 mm from the basal extreme. Microscopic examination of nuclei in damaged OHCs revealed two distinct morphological alterations, nuclear condensation or nuclear swelling. Nuclear condensation 


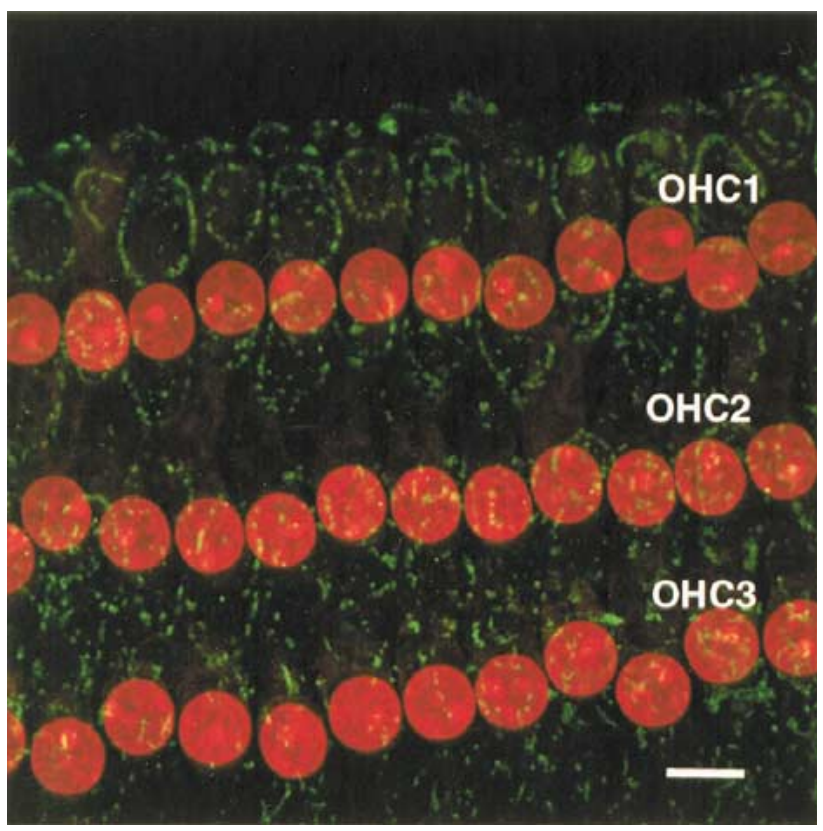

FIG. 1. Immunohistological labeling of cytochrome $\mathrm{c}$ in a normal cochlea without exposure to noise. The red DNA intercalating fluorophore, $\mathrm{PI}$, was used to label $\mathrm{OHC}$ nuclei. The weak oval, green fluorescence is used to localize cytochrome $\mathrm{c}$ in the cytoplasm along the inner surface of the plasma membrane where mitochondria normally reside. Scale bar $=6 \mu \mathrm{m}$.

is associated with apoptosis, whereas nuclear swelling is a characteristic of necrosis (Majno and Joris 1995).

A control cochlea in Figure 2A indicates no active caspase-3 present. Figure 2B illustrates the spatial correlation between active caspase- 3 in the cytosol with an OHC marked by an apoptotic nuclear morphology. Activation of caspase-3 typically takes place only in the OHCs associated with condensed nuclei. In order to eliminate the possibility for overlap of fluorescence derived from caspase labeling of adjacent apoptotic cells, we chose a less severely damaged section of the organ of Corti whereby only one OHC nucleus reveals an apoptotic morphology. Notice that only the OHC having a condensed nucleus also shows positive caspase-3 staining (arrow in Fig. 2B). In contrast, the $\mathrm{OHC}$ with a swollen nucleus and viable OHCs do not exhibit fluorescence indicative of active caspase (arrow in Fig. 2C). The spatial correlation between active caspase- 3 and nuclear condensation clearly indicates that caspase-3 activation is specific only for apoptotic OHCs. It should be noted that the pixel intensity in Figure $2 \mathrm{C}$ is enhanced relative to the other figures in order to provide better contrast with the weakly stained necrotic nuclei.

Activation of caspase-3 in cochleas has been reported in a previous study and found to take place immediately following noise exposure $(\mathrm{Hu}$ et al. 2002a). Here we focus on the duration of activated caspase- 3 in the cochleas collected at $6 \mathrm{~h}$ and 2 days
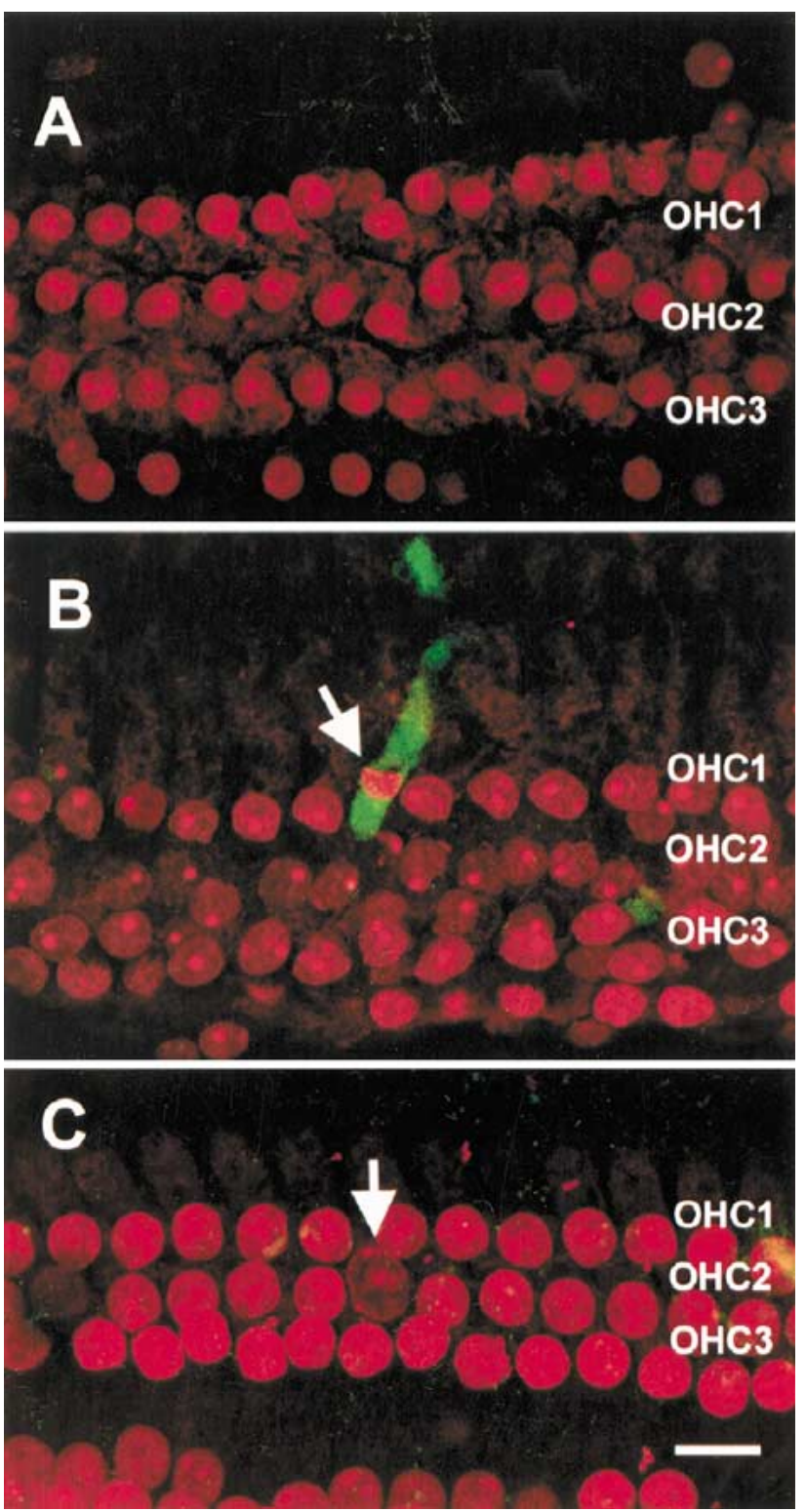

FIG. 2. Images of cochleas stained for activated caspase-3 and with PI without exposure to noise and noise-exposed cochleas stained immediately after exposure. A. The normal cochlea does not exhibit a green fluorescence indicative of caspase-3 activity in OHCs. B. Caspase-3 labeling in an apoptotic $\mathrm{OHC}$ from a cochlea exposed to noise. Notice that there is a strong green fluorescence in the region immediately surrounding the $\mathrm{OHC}$ having a condensed nucleus (arrow), whereas no fluorescence is present in the neighboring viable OHCs. C. Caspase-3 labeling of a necrotic OHC (arrow). Notice that neither this $\mathrm{OHC}$ with a swollen nucleus, nor any of the normal $\mathrm{HC}$ nearby, exhibits any caspase-3 labeling. Scale bar $=10 \mu \mathrm{m}$.

after noise exposure. Activated caspase- 3 persisted in the OHCs that also exhibited condensed or fragmented nuclei at both $6 \mathrm{~h}$ and 2 days after exposure to noise. Generally, the number of OHCs that exhibit caspase- 3 activation correlated with the overall extent of noise damage to the organ of Corti. That is, the greater the number of OHCs having condensed nuclei, the greater the number of OHCs showing posi- 


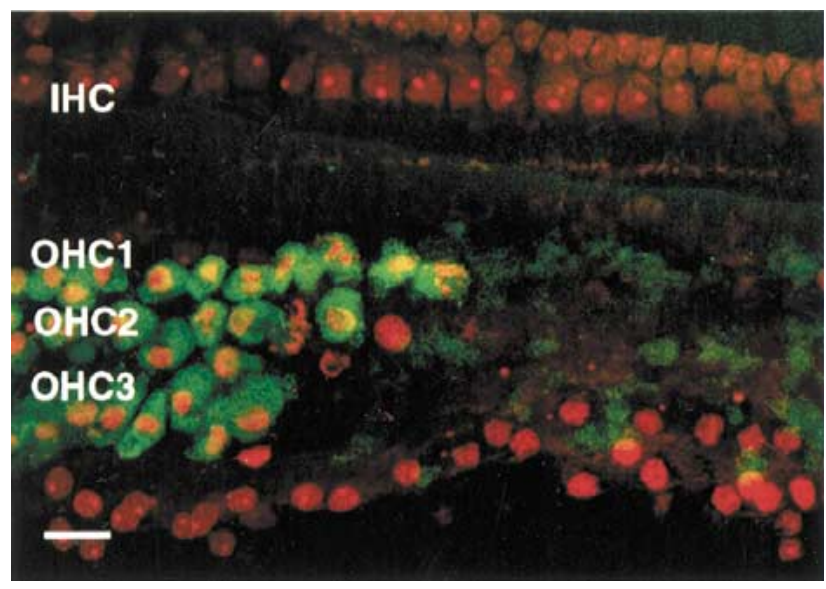

FIG. 3. Image illustrating caspase-3 labeling and with PI in the organ of Corti in a large lesion collected 2 days after noise exposure. On the left side of the image, where most of the nuclei appear shrunken and irregular in shape, there is increased PI fluorescence along with positive caspase-3 labeling in these apoptotic OHCs. On the right side of the image, virtually all of the $\mathrm{OHC}$ nuclei have disappeared. However, there is a trace of caspase-3 fluorescence remaining in this area. Scale bar $=15 \mu \mathrm{m}$.
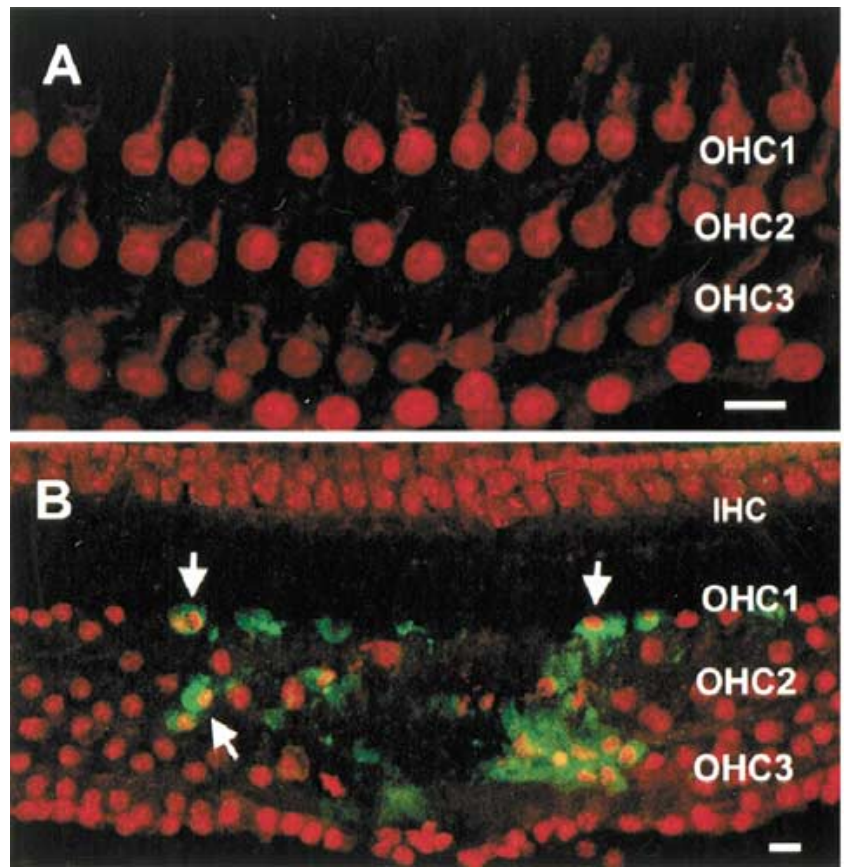

FIG. 5. Images illustrating fluorescence associated with either activated caspase- 8 or caspase- 9 in HCs from normal cochleas or cochleas exposed to noise. Neither caspase-9 (A) nor caspase-8 (C) exhibits activity in normal, unexposed cochleas and there is a lack of detectable green fluorescence. B. Caspase- 9 staining in noise-exposed cochlea. In this area, there is a focus of damage in the organ of

tive caspase- 3 staining. In addition, the caspase-3positive OHCs were found more frequently in the basal part of the organ of Corti, which is in agreement with the finding that this area possessed the greatest number of apoptotic OHCs.

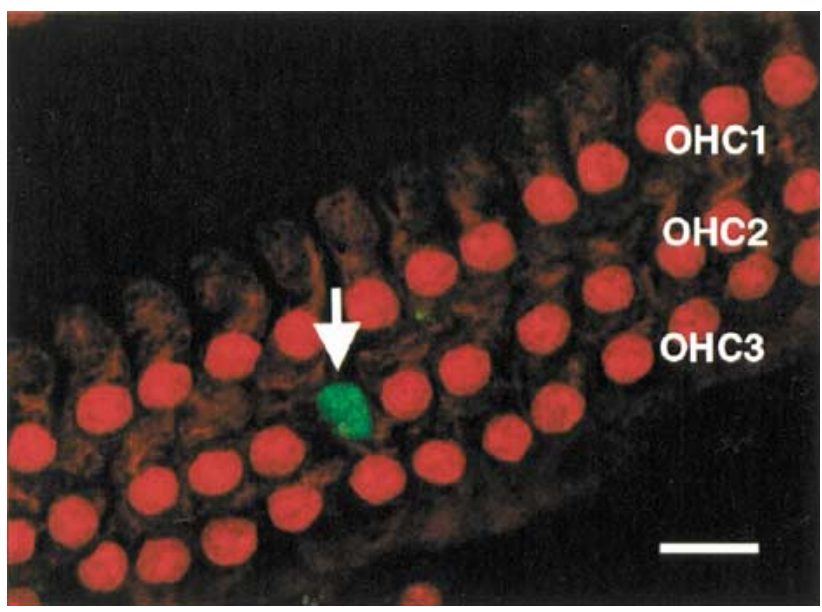

FIG. 4. An image within the organ of Corti showing a confined area with green, caspase-3 fluorescence. Notice that this area (arrow) exhibits very little if any red fluorescence, suggesting that the nucleus from this $\mathrm{OHC}$ has been nearly completely disintegrated. Scale bar $=10 \mu \mathrm{m}$.
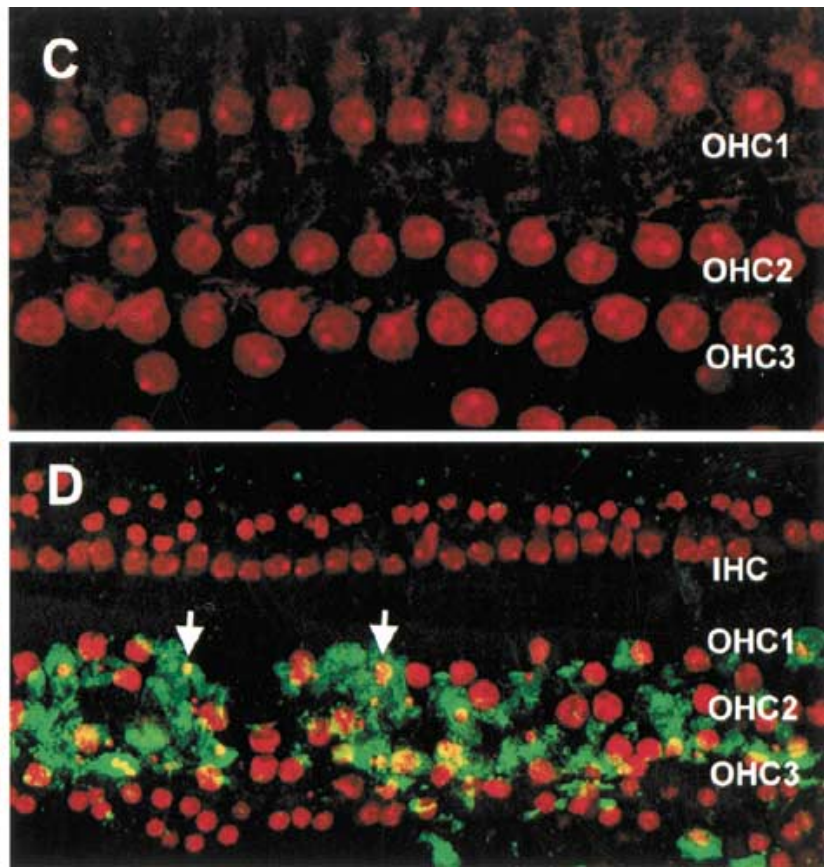

Corti. Notice that only OHCs having condensed nuclei show caspase-9 labeling (arrows). D. Caspase-8 staining in noise-exposed cochlea. Similar to caspase-9 staining, caspase-8 fluorescence also appeared in the OHCs with condensed or fragmented nuclei (arrows). Scale bar $=25 \mu \mathrm{m}$.

Figure 3 illustrates caspase-3 labeling in the organ of Corti exhibiting a large lesion collected 2 days after noise exposure. On the left side of the image, most of the OHCs are apoptotic, as evidenced by both their morphology and caspase-3 fluorescence. The nuclei 

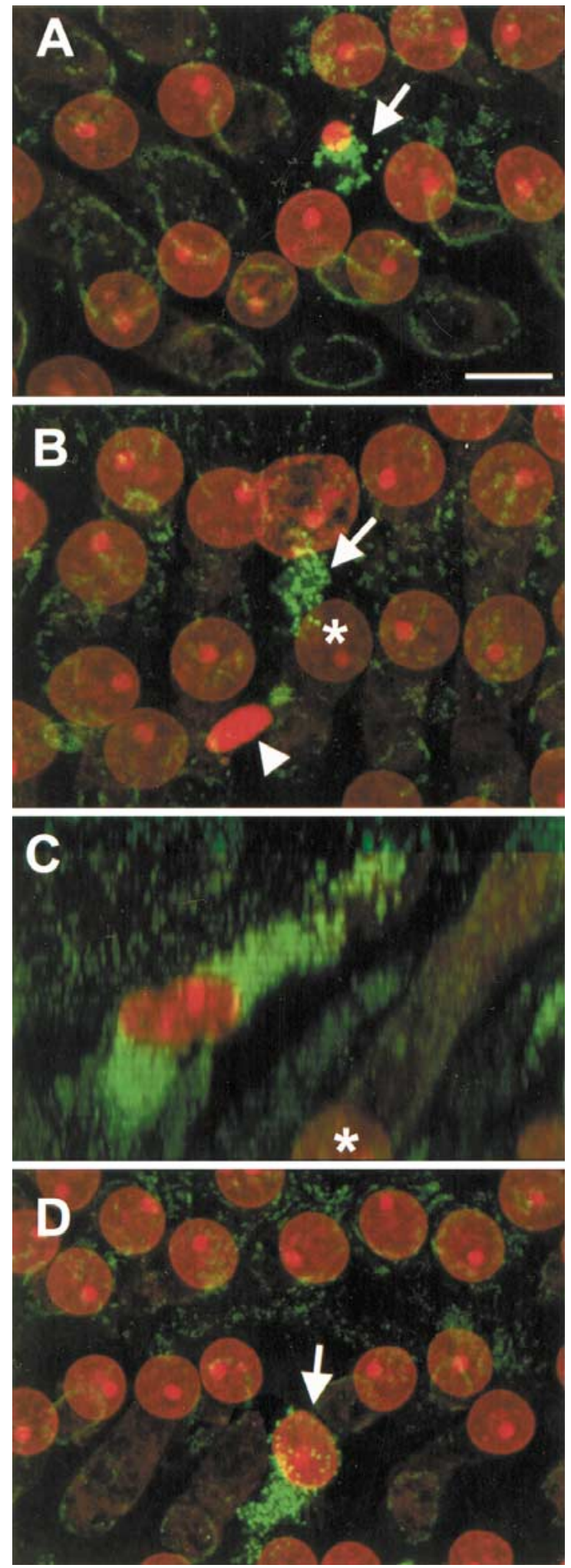

appear shrunken and irregular in shape and there is increased PI fluorescence. On the right side of the image, virtually all of the OHC nuclei have disappeared. However, there is a residual caspase-3 fluorescence in this area. The persistence of caspase- 3
FIG. 6. Images showing cytochrome c release from $\mathrm{HC}$ following noise exposure. A. Immediately after noise exposure, a punctate fluorescence is formed around an apoptotic nucleus (arrow), indicating the release of mitochondrial cytochrome c into the cytosol. This fluorescence pattern is representative of apoptotic cells and differs from that of neighboring viable $\mathrm{OHCs}$ which demonstrate a weak fluorescent circle inside the plasma membrane. B. Punctate fluorescence also appears around a swollen nucleus (arrow), indicating that release ofcytochrome c also occurs in the necrotic cells. The arrowhead indicates a condensed nucleus. The green, punctate fluorescence surrounding this nucleus was eliminated in order to show the fluorescence associated with the nearby necrotic cell directly above. In order to clearly illustrate the anatomic relationship between the released cytochrome $\mathrm{c}$ and the swollen nuclei, the images that were used to generate image $\mathbf{B}$ were reprocessed to form a side-view image $(\mathbf{C})$. Notice that the released cytochrome $\mathrm{c}$ is located in the $\mathrm{HC}$ having the swollen nucleus and not in the neighboring $\mathrm{OHC}$ nuclei (asterisk in B and C) at a considerable distance below. D. The release of cytochrome $\mathrm{c}$ is also shown to take place in an $\mathrm{OHC}$ having a relatively normal nucleus (arrow). Scale bar $=4 \mu \mathrm{m}$.

activity during apoptosis is more clearly demonstrated in Figure 4 where damage to the OHCs is mild. Notice that an OHC (arrow) with a totally disintegrated nucleus retains caspase-3 fluorescence within the confines of the cell body.

\section{Detection of activated caspases- 8 and -9}

Figures $5 \mathrm{~A}$ and $\mathrm{C}$ demonstrate a lack of fluorescence associated with active caspase- 9 and -8 , respectively, in control cochleas i.e., without noise exposure. Fluorescence labeling following noise exposure shows that activated caspase- 8 or caspase- 9 appears only in OHCs having condensed nuclei in a fashion similar to caspase-3. Figure 5B shows a cochlea stained for caspase-9 immediately after noise exposure. In this area, there is a focus of damage to OHCs. At the very center of the damage, many OHC nuclei have disappeared and many OHC nuclei remaining in the marginal regions are condensed. Notice that only OHCs having condensed nuclei show caspase-9 labeling (arrows). Figure 5D shows caspase-8 fluorescence in a cochlea collected immediately after noise exposure. Caspase- 8 fluorescence also appeared in OHCs having condensed or fragmented nuclei in a fashion similar to caspase-9 labeling. Since all three caspases are found to be active at the earliest stages of our analysis using the described protocol, it was not possible to discern the sequence of caspase activation.

\section{Release of cytochrome c}

The spatial relationship between nuclear changes and translocation of cytochrome $\mathrm{c}$ to the cytosol can be obtained by analyzing a complete set of 3-dimensional images collected by confocal microscopy. 

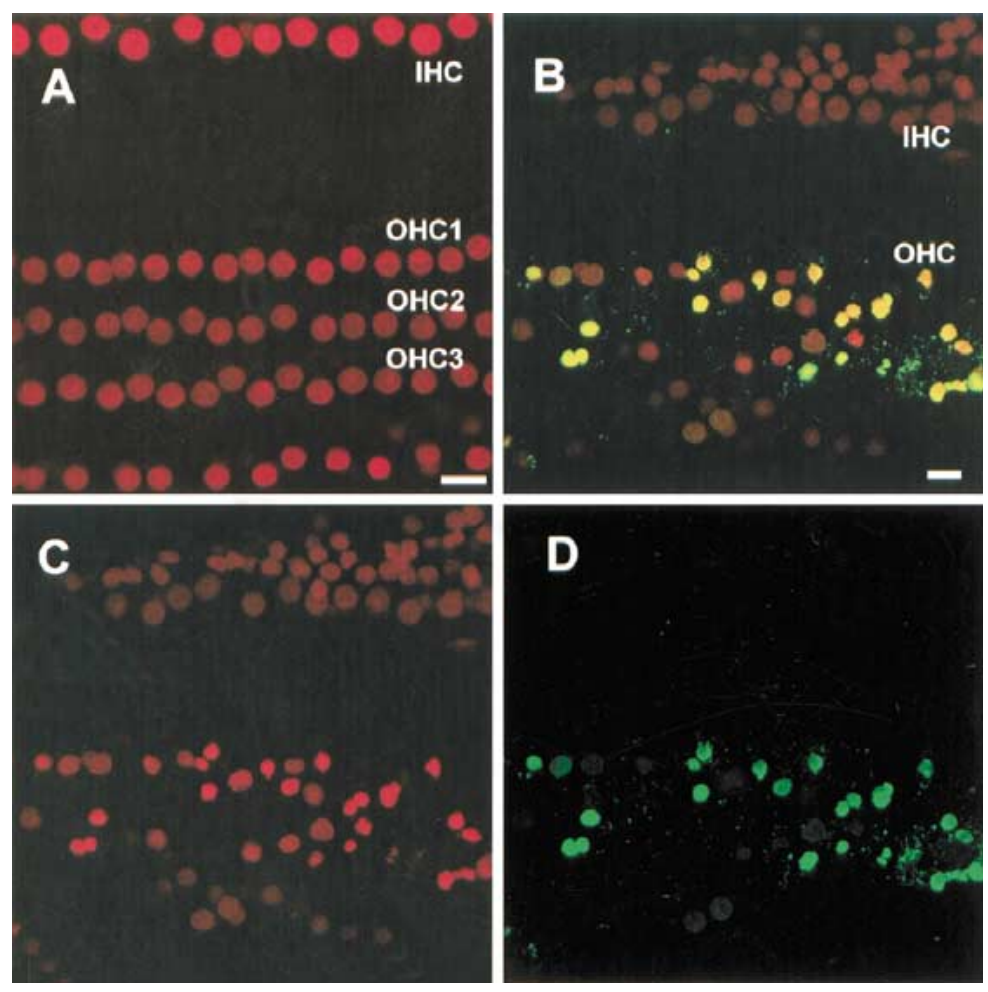

FIG. 7. Images showing TUNEL staining of HCs in a control and in a noise-damaged cochlea. A. TUNEL labeling in the control cochlea. These normal HC nuclei appear uniformly spaced and have no associated TUNEL staining. B. Combined TUNEL/PI staining in a cochlea exposed to noise. The TUNEL-positive nuclei appear yellow because of the combination of green florescence on the red fluorescence (PI) in the apoptotic HCs. To better illustrate this double staining procedure, the red PI fluorescence and green TUNEL fluorescence are presented separately. C. PI labeling indicates two cell populations, one with enlarged, more diffusely labeled nuclei that are necrotic and a second with condensed and more intenselylabeled nuclei that are apoptotic. D. Notice that all condensed nuclei exhibit green TUNEL fluorescence, indicating that DNA fragmentation is taking place in these apoptotic cells.
However, due to malformation and/or displacement of cell bodies from dying cells, the projected 2-D image obtained from a composite of individual slices often appears confusing in severely damaged sections of the organ of Corti. In order to minimize interference from neighboring OHCs and to more clearly illustrate the correlation between nuclear alterations and cytochrome $\mathrm{c}$ translocation within individual cells, we selected images showing only the less severely damaged areas of the organ of Corti. In this manner, it is possible to discern the fluorescence patterns associated with individual OHCs undergoing cell death.

Figure 6 shows four sections of the organ of Corti from a cochlea collected immediately after noise exposure. In order to highlight cytochrome c translocation to the cytoplasm in the area above the nuclear region, cytochrome $\mathrm{c}$ fluorescence in the subnuclear region is present but not shown. Notice that the fluorescence intensity for cytochrome $\mathrm{c}$ was noticeably increased in the apoptotic OHC having a shrunken nucleus (arrow in Fig. 6A). In addition, a cluster of punctate fluorescence was noted in the cytoplasm rather than the circular staining pattern in the adjacent, viable OHCs. Figure 6B shows that translocation of cytochrome c also takes place in an OHC having a swollen nucleus as indicated by an arrow. Thus, necrotic cells exhibit a punctate fluorescence indicating cytochrome $\mathrm{c}$ release in a pattern similar to that of condensed, apoptotic nuclei. In Figure 6B, there is a nearby $\mathrm{OHC}$ with a condensed nucleus (arrowhead) where the cytochrome $\mathrm{c}$ fluorescence has been excluded from the projected image in order to minimize interference when observing the $\mathrm{OHC}$ with the swollen nucleus. Figure $6 \mathrm{C}$ is a side view of the same necrotic OHC shown in Figure $6 \mathrm{~B}$ with cytochrome $\mathrm{c}$ fluorescence present inside the cell body both above and below the nucleus. Present at the bottom of Figure 6C (asterisk) is the same normal-appearing nuclei from Figure 6B (asterisk) and located at a considerable distance below the cytochrome $\mathrm{c}$ fluorescence.

Figure 6D shows that an $\mathrm{OHC}$ having punctate fluorescence, indicating cytochrome $\mathrm{c}$ release from mitochondria, is clearly noted prior to any nuclear changes. Therefore, at this stage it is not possible to determine the death pathway taken by this cell based on nuclear morphology alone. However, release of cytochrome $\mathrm{c}$ is dramatic in this cell, suggesting that cytochrome c release is one of the early events in the cell-damaging process. This observation, coupled with the early activation of caspase-9, supports the notion that mitochondrial changes may precede other apoptotic signaling events and is consistent with reports that redistribution of cytochrome $c$ is an early apoptotic event in certain cell types (Dinsdale et al. 1999).

\section{DNA fragmentation assay}

Noise controls consisted of cochleas treated with PI (red) and TUNEL reagents (green) but without noise 
exposure and are depicted in Figure 7A. Control OHC nuclei are regularly spaced, evenly stained with PI and without evidence of DNA degradation. In noise-exposed cochleas, a lesion containing both yellow- and red-labeled nuclei are noted (Fig. 7B). The majority of these damaged and displaced nuclei are yellow, providing an indication that DNA fragmentation is taking place and representative of apoptotic nuclei. The remaining nuclei are enlarged, permeable to PI (red), and indicative of necrosis. Figures $7 \mathrm{C}$ and $\mathrm{D}$ are images of nuclei using either a red or a green activating laser, respectively. Figure $7 \mathrm{C}$ clearly contrasts the more intensely colored and shrunken (apoptotic) nuclei from the more diffusely labeled and enlarged (necrotic) nuclei. Addition of TUNEL reagents and activation by a green laser source identifies only the apoptotic nuclei (Fig. 7D) as they undergo DNA degradation and provides an additional means of detecting apoptotic nuclei. Figure 7B clearly associates the red shrunken nuclei with the green TUNEL-labeled nuclei to yield yellow nuclei. This combination of staining methods also provides a more robust validation of our initial observation that apoptosis and necrosis are simultaneously taking place in response to noise.

\section{DISCUSSION}

In our study we detected activation of three members of the caspase family, caspase- $-3,-8$, and -9 , in the OHCs of chinchilla cochleas exposed to intense noise. We also examined the release of mitochondrial cytochrome $\mathrm{c}$ in the noise-damaged OHCs. The significant findings of this study are as follows: (1) In apoptotic OHCs as featured by nuclear condensation or fragmentation, caspase-3 was activated, whereas in necrotic OHCs characterized by nuclear swelling, there was no caspase-3 activation. Once caspase-3 was activated, its activity remained high in apoptotic OHCs well after the apoptotic nuclei were totally disintegrated. (2) Both caspases- 8 and -9 were activated after exposure to noise. Activation of caspases- 8 and -9 occurred only in apoptotic OHCs in a pattern similar to that of caspase-3 activation. (3) Release of cytochrome c from mitochondria took place in both necrotic and apoptotic OHCs. Also, cytochrome c release in OHCs appeared to take place at an early stage of the cell death process. (4) Apoptotic and necrotic cells appear in approximately equal numbers and are easily distinguished using a combination of TUNEL/PI labeling methodology.

The essential role of caspase-3 in the killing process of the apoptotic cell has been recognized in a multitude of different cell lines and under various pathological conditions. It has been noted that acti- vated caspase- 3 targets the degradation of numerous intracellular and extracellular proteins that are essential for maintaining normal cell function. In a previous study (Hu et al. 2002b), cleavage of F-actin, an important cytoskeletal protein, was found in apoptotic OHCs. F-actin cleavage was temporarily inhibited by pretreating cochleas with a caspase-3 inhibitor. In other studies, caspase-3 has been reported to be responsible for mediating DNA fragmentation by endonucleases in apoptotic cells (Cohen 1997; Nicholson and Thornberry 1997).

It has been noted that the time required for activation of caspase-3 is associated with specific experimental conditions, such as the nature of apoptotic stimuli and cell line used. The time required for caspase activation induced in the brain either by ischemia (Han et al. 2000; Sasaki et al. 2000; Velier et al. 1999) or chemical stimuli (Kondratyev and Gale 2000; Asakura et al. 1999; Hayami et al. 1999) ranges from several hours to one day after exposure to an apoptotic stimulus. In our study, we found that activation of caspase-3 took place either shortly after noise exposure or possibly during noise exposure. Considering that (1) activation of caspase-3 was found in the processed cochlea immediately after noise exposure and that (2) the total length of time $(2.5 \mathrm{~h}$ ) required for completing the experimental manipulation, it is likely that activation of caspase-3 occurs within $2.5 \mathrm{~h}$ after noise exposure is initiated. This estimated time frame is based on the length of time required for complete processing of cochleas, which includes the noise exposure, the surgical procedure for the perfusion of caspase-3 probe, and the subsequent incubation period. The time required for caspase activation in apoptotic OHCs is unusually brief and may be a consequence of the high-intensity noise utilized. A low-intensity noise may require a longer time period for caspase-3 activation and requires further investigation.

Examination of OHC nuclei in this study, as well as in a previous study (Hu et al. 2002a), revealed a rapid development of OHC pathologies during high level noise exposure. Immediately after exposure to noise, apoptotic nuclei appeared while others were missing in damaged sections of the cochlea, particularly at the damaged center. Also, at this time point, F-actin associated with the cuticular plates of apoptotic OHCs show sections of discontinuity that are indicative of breakdown of F-actin (Hu et al. 2002b). These early changes in F-actin breakdown coincided with rapid activation of caspase- 3 and its inhibition prevented Factin cleavage. These results clearly indicate the involvement of caspase-3 in OHC apoptosis induced by exposure to intense noise. Activated caspase- 3 initiates the hydrolytic breakdown of intracellular and extracellular proteins and, consequently, the com- 
plete degradation of the apoptotic cell takes place in a relatively short time.

In addition to its rapid activation, caspase- 3 activity persists in dying OHCs well after nuclei are degraded. However, the ultimate fate of apoptotic OHCs is not as yet clear. Apoptotic bodies are generally formed, which consist of nuclear fragments and intact organelles enclosed by a plasma membrane fragment. After their formation, these apoptotic bodies are subsequently engulfed by neighboring cells or macrophages and cleared from the lesion (Nishikawa et al. 1998; Rubartelli et al. 1997). Fredelius and RaskAndersen (1990) reported that macrophages are present in the organ of Corti and survive for five days after noise exposure. Our study did not identify any obvious presence of macrophages within the organ of Corti or any sign of engulfment of apoptotic bodies by supporting cells after noise exposure. A more careful detection of macrophages, such as antibody recognition of surface antigens, is required to confirm their presence in the cochlea and to further characterize the mechanism(s) by which apoptotic bodies are removed from the cochlea. It is possible that caspase- 3 remains activated until the cell is either sufficiently degraded or the remnants removed by scavenger cells.

Activation of caspase- 3 is a downstream event in the apoptotic cascade, which can be triggered by a variety of extracellular or intracellular signaling pathways. To date, two pathways, the cell death receptor-mediated pathway and the mitochondrial pathway, have been well characterized in many cell lines to converge on caspase-3 activation. In the receptor-mediated pathway, ligands such as Fas or tumor necrosis factor $\alpha$ bind to its receptor, causing receptor aggregation and recruitment of death adapter molecules on the cytoplasmic side of the membrane (Ashkenazi and Dixit 1998). Procaspase- 8 is recruited to the complex and cleaved to form activated caspase- 8 . Activated caspase- 8 , in turn, cleaves procaspase- 3 and activates it. In the mitochondrial pathway, a proapoptotic member of the bcl-2 family, such as bax or bid, associates with the mitochondrial pore complex and directs the dissociation and the eventual release of cytochrome $c$ to the cytosol where it can associate with another key factor, Apaf-1. Apaf-1 binds cytochrome $\mathrm{c}$, dATP, or ATP and forms a large multimeric complex, which includes caspase-9. Caspase-9 is activated upon binding to Apaf-1, whereby this complex processes procaspase- 3 to its active form.

In noise-induced apoptosis, information is not as yet available as to which pathways are responsible for the activation of caspase- 3 . We therefore examined the activation of both caspases- 8 and -9 in response to noise. We found that both caspase- 8 and caspase- 9 were activated in apoptotic OHCs following noise exposure in a pattern similar to caspase-3 activation. Neither of these caspases was found in an activated state in necrotic OHCs. A more detailed analysis of the temporal sequence for activation of caspases- 8 and -9 using a concurrent fluorescence labeling procedure in noise-damaged cochleas was not possible due to the similarity in spectra of the fluorescent probes used in this study. Attempts to reduce the noise intensity yielded an inconsistent apoptotic response that precluded analysis. Therefore, it is possible that individual OHCs underwent apoptosis either through the caspase- 8 or caspase- 9 pathway alone. However, this possibility appears unlikely because probes for both caspases- 8 and -9 fluoresce in virtually all of the OHCs with condensed or fragmented nuclei. Dual staining of caspases- 8 and -9 using different fluorophores could yield a more definitive outcome as to their activation sequence.

Understanding the relative contribution of caspases- 8 and -9 and their activation sequence are important issues that should be pursued. For instance, caspase- 8 activation could provide information regarding a contribution from ischemia/reperfusion and subsequent signaling through cell death receptor pathways, whereas caspase- 9 could indicate a contribution of mitochondrial overstimulation. Unfortunately, additional experiments yielded little useful information. Use of specific, nonfluorescent inhibitors for either caspase- 8 or caspase- 9 did not significantly reduce caspase- 3 activation, and a pan-caspase inhibitor only slowed the apoptotic process but did not eliminate it (data not shown). One possible explanation for this data is that not all activated caspases have been identified in response to noise stimulation. We are currently testing whether, in fact, additional caspases are being activated by noise.

Translocation of cytochrome $\mathrm{c}$ from the mitochondria has been reported to take place in many cell systems during apoptosis and its release is followed by the activation of caspase-9, which, in turn, activates caspase-3 (Cai et al. 1998). The mechanism for the translocation of cytochrome $\mathrm{c}$ to the cytosol is not completely understood. Whether mitochondrial swelling and consequent disruption of the outer membrane permits the release of cytochrome c, or whether bcl-2 family members regulate cytochrome c release through their capacity to form membrane channels, is currently being debated. What is clear, however, is that release of cytochrome $\mathrm{c}$ is closely associated with apoptosis (Zhivotovsky et al. 1998). In contrast to these reports, our observation is that the release of cytochrome $\mathrm{c}$ was associated with both apoptotic and necrotic OHCs. The biological significance of this finding is not yet clear. Furthermore, release of cytochrome $\mathrm{c}$ was also noted in OHCs that demonstrated no clear indication of either necrotic 
or apoptotic nuclear morphology. This observation is in agreement with a previous report indicating that, in some cells, cytochrome c release is an early event that precedes caspase activation and that caspase inhibition prevents ultrastructural changes within the cell (Dinsdale et al. 1999). An interesting possibility is that release of cytochrome $\mathrm{c}$ in hair cells may be a common early step that triggers cell death processing. Several factors are known to contribute to the particular cell death pathway selected; these include the level of caspases activated, the energy available in the form of ATP (Leist et al. 1997), and the nature and severity of the insult (Bonfoco et al. 1995).

Evidence is accumulating that inhibition of caspase-dependent apoptosis in many cases leads to a necrotic-like cell death (Kitanaka and Kuchino 1999). One recent study shows that the apoptotic pathway is associated with massive release of activated caspases into the media, whereas in necrotic cells only inactive procaspases were detectable (Denecker et al. 2001). In the cochlea, apoptosis and necrosis appear to be intertwined in their own unique fashion in a process that is only now beginning to be uncovered. What is clear is that caspases are indispensable as initiators and effectors of the apoptotic cell death program, whereas cytochrome $\mathrm{c}$ release is associated with both necrosis and apoptosis. To this end, we have incorporated the use of multiple markers which permits the facile discrimination between apoptotic and necrotic cells within the cochlea. The morphological changes, including cytoplasmic swelling and plasma membrane permeabilization as identified by the incorporation of PI, can be used to clearly distinguish necrotic cells, whereas apoptotic cells are readily identifiable by the formation of apoptotic bodies in combination with either DNA degradation or the release of activated caspases. These differences will serve as important markers in the stepwise characterization of the hair cell death responses to noise.

\section{ACKNOWLEDGMENTS}

This research was supported by grant NICDC1R21 DC04984-01.

\section{REFERENCES}

Adrain C, Martin SJ. The mitochondrial apoptosome: a killer unleashed by the cytochrome seas. Trends Biochem. Sci. 26:390397, 2001.

Asakura T, Sawai T, Hashidume Y, Ohkawa Y, Yokoyama S, Ohkawa K. Caspase-3 activation during apoptosis caused by glutathionedoxorubicin conjugate. Br. J. Cancer 80:711-715, 1999.

Ashrenazi A, Dixit VM. Death receptors: signaling and modulation. Science 281:1305-1308, 1998.
Bonfoco E, Krainc D, Ankarcrona M, Nicotera P, Lipton SA. Apoptosis and necrosis: two distinct events induced, respectively, by mild and intense insults with $N$-methyl-D-aspartate or nitric oxide/superoxide in cortical cell cultures. Proc. Natl. Acad. Sci. USA 92:7162-7166, 1995.

CAI J, YANG J, Jones DP. Mitochondrial control of apoptosis: the role of cytochrome c. Biochim. Biophys. Acta 1366:139-149, 1998.

ChEN YR, TAN TH. The c-Jun N-terminal kinase pathway and apoptotic signaling [review]. Int. J. Oncol. 16:651-662, 2000.

CoHen GM. Caspases: the executioners of apoptosis. Biochem. J. 326:1-16, 1997.

Denecker G, Vercammen D, Steemans M, Vanden Berghe T, Brouckaert G, Van Loo G, Zhivotovsky B, Fiers W, Grooten J, Declerce W, Vandenabeele P. Death receptor-induced apoptotic and necrotic cell death: differential role of caspases and mitochondria. Cell Death Differ. 8:829-840, 2001.

Dinsdale D, Zhuang J, Cohen GM. Redistribution of cytochrome c precedes the activation of caspase-dependent formation of ultracondensed mitochondria, with a reduced inner membrane potential in apoptotic moncytes. Am. J. Pathol. 155:607-618, 1999.

EldADAh BA, FAdEn AI. Caspase pathways, neuronal apoptosis, and CNS injury. J. Neurotrauma 17:811-829, 2000.

Fredelius L, Rask-Andersen H. The role of macrophages in the disposal of degeneration products within the organ of corti after acoustic overstimulation. Acta Otolaryngol. (Stockh.) 109:76-82, 1990.

Han BH, D'Costa A, Back SA, Parsadanian M, Patel S, Shah AR, Gidday JM, Srinivasan A, Deshmukh M, Holtzman DM. BDNF blocks caspase-3 activation in neonatal hypoxia-ischemia. Neurobiol. Dis. 7:38-53, 2000.

Hatefi Y. The mitochondrial electron transport and oxidative phosphorylation system. Annu. Rev. Biochem. 54:1015-1069, 1985.

Hayami S, Ikeda K, Sun F, Tanaka K, Kojo S. Increase of caspase-3 activity in rat liver and plasma by thioacetamide. Biochem. Pharmacol. 58:1941-1943, 1999.

Henderson D, Hamernik RP. Impulse noise: critical review. J. Acoust. Soc. Am. 80:569-584, 1986.

Hu BH, Guo W, Wang PY, Henderson D, Jiang SC. Intense noiseinduced apoptosis in hair cells of guinea pig cochleae. Acta Otolaryngol. (Stockh.) 120:19-24, 2000.

Hu BH, Nicotera T, Henderson D. Involvement of apoptosis in progression of cochlear lesion following exposure to intense noise. Hear. Res. 166(1-2):62-71, 2002a.

Hu BH, Nicotera T, Henderson D. F-actin cleavage in apoptotic outer hair cells in chinchilla cochleas exposed to intense noise. Hear. Res. 172(1-2):1-9, 2002b.

Kitanaka C, Kuchino Y. Caspase-independent programmed cell death with necrotic morphology. Cell Death Differ. 6:508-515, 1999.

Kondratyev A, Gale K. Intracerebral injection of caspase-3 inhibitor prevents neuronal apoptosis after kainic acid-evoked status epilepticus. Brain Res. Mol. Brain Res. 75:216-224, 2000.

Leist M, Single B, Castoldi AF, Kuhnle S, Nicotera P. Intracellular adenosine triphosphate (ATP) concentration: a switch in the decision between apoptosis and necrosis. J. Exp. Med. 185:1481-1486, 1997.

Lim DJ, Melnick W. Acoustic damage of the cochlea. A scanning and transmission electron microscopic observation. Arch Otolaryngol. 94:294-305, 1971.

Lo AC, Houenou LJ, Oppenheim RW. Apoptosis in the nervous system: morphological features, methods, pathology, and prevention. Arch Histol. Cytol. 58:139-149, 1995.

Maher P, Schubert D. Signaling by reactive oxygen species in the nervous system. Cell Mol. Life Sci. 57:1287-1305, 2000. 
Majno G, Joris I. Apoptosis, oncosis, and necrosis. An overview of cell death. Am. J. Pathol. 146:3-15, 1995.

MiLLER DK. The role of the Caspase family of cysteine proteases in apoptosis. Semin. Immunol. 9:35-49, 1997.

Nicholson DW, Thornberry NA. Caspases: killer proteases. Trends Biochem. Sci. 22:299-306, 1997.

Nicotera T, Henderson D, Hu BH, Zheng XY. Noise exposure and mechanisms of hair cell death. In: Prasher D, Henderson D, Kopke R, Salvi R, Hamernik R (Eds.) Noise Induced Hearing Loss: Advances in Basic Science and Clinical Application. NRN Publications, Cambridge, England, 2002.

Nishikawa A, Murata E, Akita M, Kaneko K, Moriya O, Tomita M, HAYASHI H. Roles of macrophages in programmed cell death and remodeling of tail and body muscle of Xenopus laevis during metamorphosis. Histochem. Cell Biol. 109:11-17, 1998.

Oмata T, Schatzle W. Electron microscopical studies of the effect of time lapse on outer hair cells in acoustically exposed rabbits. Acta Otolaryngol. (Stockh.) 92:211-220, 1981.

Pirvola U, Xing-Qun L, Virkkala J, Saarma M, Murakata C, Camoratto AM, WaLton KM, YLIKoski J. Rescue of hearing, auditory hair cells, and neurons by CEP-1347/KT7515, an inhibitor of cJun N-terminal kinase activation. J. Neurosci. 20:43-50, 2000.

Rubartelli A, Poggi A, Zocchi MR. The selective engulfment of apoptotic bodies by dendritic cells is mediated by the alpha(v)beta3 integrin and requires intracellular and extracellular calcium. Eur. J. Immunol. 27:1893-1900, 1997.

Sasaki C, Kitagawa H, Zhang WR, Warita H, Sakai K, Abe K. Temporal profile of cytochrome $\mathrm{c}$ and caspase-3 immunoreactivities and TUNEL staining after permanent middle cerebral artery occlusion in rats. Neurol. Res. 22:223-228, 2000.

Strasser A, O'Connor L, Dixit VM. Apoptosis signaling. Annu. Rev. Biochem. 69:217-245, 2000.

Tаканалні A. Caspase: executioner and undertaker of apoptosis. Int. J. Hematol. 70:226-232, 1999.

Velier JJ, Ellison JA, Kikly KK, Spera PA, Barone FC, Feuerstein GZ. Caspase- 8 and caspase- 3 are expressed by different populations of cortical neurons undergoing delayed cell death after focal stroke in the rat. J. Neurosci. 19:5932-5941, 1999.

Zhivotovsky B, Orrenius S, Brustugun OT, Doskeland SO. Injected cytochrome c induces apoptosis. Nature 391:449-450, 1998. 